\title{
Valores hematológicos del armadillo peludo (Chaetophractus nationi) en cautiverio en un parque zoológico de Lima, Perú
}

\author{
(Hematological values of the hairy armadillo (Chaetophractus nationi) \\ in captivity at one Zoological Park Lima, Perú)
}

\author{
E. Solano Bravo*
}

http://dx.doi.org/10.21503/CienciayDesarrollo.2011.v14.06

\section{RESUMEN}

El objetivo del presente estudio fue determinar los valores hematológicos del armadillo peludo (Chaetophractus nationi) en cautiverio en un parque zoológico. Su distribución geográfica se ubica entre los 3500 y 4300 m.s.n.m., en los arenales de las mesetas andinas de Perú, Argentina, Bolivia y Chile. En la actualidad, su situación es vulnerable según la Unión Internacional para la Conservación de la Naturaleza (UICN). Siete armadillos adultos aparentemente sanos fueron sometidos a un estudio hematológico con el fin de determinar parámetros referenciales en esta población específica. El diseño estadístico incluyó la determinación del promedio y desviación estándar. Los valores obtenidos en promedio fueron: eritrocitos $=4738571,43 / \mathrm{ul}$; leucocitos $=6064,29 / \mathrm{ul}$; hemoglobina $=13,30 \mathrm{~g} / \mathrm{dl}$; hematocrito $=36,43 \%$; neutrófilos $=60,86 \%$; linfocitos $=23,71 \%$; monocitos $=0,86 \%$; eosinófilos $=10,00 \%$; basófilos $=2,14$ $\%$ y recuento de plaquetas $=267285,71 / \mathrm{ul}$.

Palabras clave: valores hematológicos, armadillo, cautiverio, zoológico.

\section{ABSTRACT}

The purpose of the present study was to determine the hematological values of the hairy armadillo (Chaetophractus nationi) in captivity at the one Zoological Park. Its geographic distribution ranges between 3500 to 4300 m. a.s. l. in the Andean sand plates of Peru, Argentina, Bolivia y Chile. Actually its situation is vulnerable according to the International Union for Conservation of Nature (IUCN). Seven adult hairy armadillos apparently healthy were used for a hematological study in order to determinate the referential parameters for this species. The average and the standard deviation were determined Erythrocytes $=4738571,43 / \mathrm{ul}$; leucocytes $=6064,29 / \mathrm{ul}$; hemoglobin $=13,30 \mathrm{~g} / \mathrm{dl}$; hematocrit $=$ $36,43 \%$; neutrophils $=60,86 \%$; lymphocytes $=23,71 \%$; monocytes $=0,86 \%$; eosinophils $=10,00 \%$; basophiles $=2,14 \%$ and platelets $=267285,71 / \mathrm{ul}$.

Key words: hematological values, armadillo, captivity, zoo.

* En la presente investigación, el autor ha contado con la colaboración de C. Hermoza Guerra, C. Sala Lazarte y H. Samamé Beltrán. 


\section{INTRODUCCIÓN}

Los armadillos representan un antiguo y primitivo grupo de mamíferos. La situación de esta especie es vulnerable según la Unión Internacional para la Conservación de la Naturaleza (UICN) ${ }^{1}$, y los estudios tanto de su anatomía como fisiología son muy pocos. Actualmente, no se cuenta con reportes de los parámetros hematológicos para el armadillo peludo (Chaetophractus nationi). El objetivo de este estudio es determinar los valores hematológicos referenciales para el $C$. nationi mantenido en cautiverio en las instalaciones de un parque zoológico de Lima (setiembreoctubre de 2008).

\section{MATERIAL Y MÉTODOS}

La muestra sanguínea se obtuvo por punción en la vena safena lateral, previa inmovilización química de los animales, según el protocolo anestésico del zoológico. La población de $C$. nationi adultos, aparentemente sanos y mantenidos en cautiverio, fue de 7 especímenes: 5 machos y 2 hembras. Se recolectó aproximadamente $1 \mathrm{~cm}^{3}$ de sangre por cada tubo EDTA MiniCollect. Las muestras fueron llevadas a un laboratorio particular para los análisis correspondientes.

\section{RESULTADOS}

De una población de 7 armadillos adultos, aparentemente sanos y en cautiverio del zoológico, se obtuvieron los valores hematológicos (Tabla 1).

\section{DISCUSIÓN}

Los valores hematológicos obtenidos de una población de 7 especímenes de $C$. nationi de un zoológico en la ciudad de Lima fueron: eritrocitos 4738571,43 /ul, hemoglobina 13,30 g/dl y hematocrito $36,43 \%$, menores en promedio a los datos reportados para la especie D. hybridus, En cuanto, al $D$. novemcinctus, no se tiene referencias de eritrocito para esta especie en cautiverio. Por otra parte, se encontró que los valores obteni-

\section{Tabla 1. Valores hematológicos del Chaetophractus nationi, Dasypus bybridusy Dasypus novemcinctus mantenidos en cautiverio.}

\begin{tabular}{|c|c|c|c|}
\hline VALORES & ${ }^{* *}$ C. NATIONI & ${ }^{*} D . H Y B R I D U S$ & ${ }^{*} D$. NOVEMCINCTUS \\
\hline Eritrocitos /ul ${ }^{(1)}$ & $4738571,43 \pm 593426,58$ & 5980000,00 & - \\
\hline Leucocitos /ul & $6064,29 \pm 1397,12$ & 9250,00 & 8840,00 \\
\hline Hemoglobina $\mathrm{g} / \mathrm{dl}$ & $13,30 \pm 1,24$ & 16,49 & - \\
\hline Hematocrito \% & $36,43 \pm 1,92$ & 40,07 & - \\
\hline Neutrófilos \% & $60,86 \pm 9,89$ & 58,00 & 33,00 \\
\hline Linfocitos \% & $23,71 \pm 11,58$ & 33,50 & 52,00 \\
\hline Monocitos \% & $0,86 \pm 1,12$ & 4,50 & 0,70 \\
\hline Eosinófilos \% & $10,00 \pm 1,41$ & 3,00 & 4,00 \\
\hline Basófilos \% & $2,14 \pm 1,64$ & 0,50 & 0,70 \\
\hline Recuento de Plaquetas /ul & $267285,71 \pm 154430,49$ & - & - \\
\hline
\end{tabular}


dos para el $C$. nationi en cuanto a leucocitos (6 $064,29 / \mathrm{ul})$ y linfocitos $(23,71 \%)$ fueron menores en promedio a los datos reportados para el $D$. bybridus y $D$. novemcinctus en cautiverio.

El valor obtenido de monocitos fue de 0,86 $\%$ para el $C$. nationi, encontrándose que este valor fue menor en promedio a los datos reportados para las especies $D$. hybridus, y mayor en promedio a los datos reportados para el $D$. novemcinctus en cautiverio. En el caso del $C$. nationi, los valores obtenidos de neutrófilos $(60,86 \%)$, eosinófilos $(10,00 \%)$ y de basófilos $(2,14 \%)$ fueron mayores en promedio a los datos reportados para las especies $D$. bybridus y $D$. novemcinctus en cautiverio.

El valor de plaquetas obtenido para el $C . n a-$ tioni fue de 267285,71 / ul, el mismo que no pudo ser comparado porque no se tiene reportes sobre los datos de las plaquetas para las especies de D. hybridus y D. novemcinctus mantenidos en cautiverio.

Las diferencias que se observan se pueden deber a que los armadillos en estudio pertenecen al género Chaetoprhactus, mientras que el $D$. bybridus y $D$. novemcinctus pertenecen al género Dasypus. En realidad son especies distintas y con hábitos propios, ya que, el $C$. nationi habita en climas fríos, en los arenales y mesetas andinas, $3,4,5$ a diferencia del $D$. novemcinctus, que habita en Sudamérica, Centroamérica y América del Norte y tiene una gran variedad de hábitats de las regiones semiáridas, sabanas y matorrales de los bosques húmedos tropicales y de montaña, bien adaptado a ambientes modificados y a hábitats con vegetación secundaria. Estos armadillos no están presentes en el páramo o puna, o en las regiones desérticas, sino que se distribuyen desde los 2000 hasta los 3000 m.s.n.m. aproximadamente. ${ }^{1,3,4,5,6,7}$ Por su parte, el $D$. bybridus se encuentra en Argentina, Brasil, Paraguay y Uruguay, donde habita en regiones de bosque subtropical, bosque seco tropical y pastizales. ${ }^{8,9}$

\section{REFERENCIAS BIBLIOGRÁFICAS}

1. Cites. República de Bolivia. "Propuesta. Exámenes de las propuestas de enmienda a los apéndices I y II". En: http://www.cites. org/esp/cop/10/prop/S10-18.pdf. Acceso el 10 de marzo de 2009.

2. Escobar Gutiérrez A., Amescua de Bernés M. "El armadillo: un nuevo animal de experimentación para el estudio de las zoonosis". En: www.fmvz.unam.mx/fmvz/ cienciavet/revistas/CVvol3/CVv3c07.pdf. Acceso el 14 de setiembre de 2008.

3. Cardozo K. "Chaetophractus nationi. El Quirquincho”. En: http://www. revistaciencias.com/publicaciones/ EpZypupEVFAdoacvMm.php. Acceso el 5 de setiembre de 2008.

4. Arkive. "Andean Hairy Armadillo (Chaetophractus nationi)". En: http:// www.arkive.org/andean-hairy-armadillo/ chaetophractus-nationi/description.html. Acceso el 10 marzo de 2009.

5. Vizcaíno S.F., Loughry W. The Biology of the Xenarthra. Florida, University Press of Florida, 2008.

6. FAO. "Wildlife Utilization in Latin America". En: http://www.fao.org/docrep/T0750E/ t0750e0g.htm. Acceso el 26 agosto de 2009.

7. University of Michigan Museum of Zoology. Dasypusnovemcinctus. En:htt:/animaldiversity. ummz.umich.edu/site/accounts/information/ Dasypus_novemcinctus.html. Acceso el 26 agosto de 2009.

8. "Genus Dasypus. Dasypus novemcinctus". En: https://www.msu.edu/ nixonjos/armadillo/ dasypus.html. Acceso el 26 agosto de 2009.

9. Arkive. "Southern long-nosed armadillo (Dasypus bybridus)”. En: http://www. arkive.org/southern-long-nosed-armadillo/ dasypus-hybridus/range-and-habitat.html. Acceso el 26 agosto de 2009. 